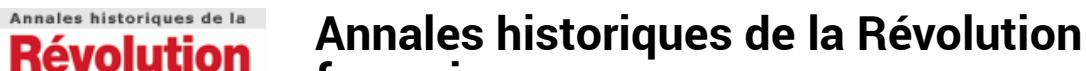

française française

368 | avril-juin 2012

Varia

\section{Thomas LE ROUX, Le laboratoire des pollutions}

industrielles. Paris, 1770-1830

\section{Bernard Gainot}

\section{(2) OpenEdition}

\section{Journals}

Édition électronique

URL : https://journals.openedition.org/ahrf/12339

DOI : 10.4000/ahrf.12339

ISSN : $1952-403 X$

Éditeur :

Armand Colin, Société des études robespierristes

Édition imprimée

Date de publication : 1 juin 2012

Pagination : 189-192

ISBN : 978-2-7489-0161-0

ISSN : 0003-4436

Référence électronique

Bernard Gainot, "Thomas Le roux, Le laboratoire des pollutions industrielles. Paris, 1770-1830 ", Annales historiques de la Révolution française [En ligne], 368 | avril-juin 2012, mis en ligne le 24 septembre 2012, consulté le 24 avril 2022. URL : http://journals.openedition.org/ahrf/12339 ; DOI : https://doi.org/ 10.4000/ahrf.12339

Ce document a été généré automatiquement le 24 avril 2022

Tous droits réservés 


\title{
Thomas LE ROUX, Le laboratoire des pollutions industrielles. Paris, 1770-1830
}

\author{
Bernard Gainot
}

\section{RÉFÉRENCE}

Thomas LE ROUX, Le laboratoire des pollutions industrielles. Paris, 1770-1830, Paris, Albin Michel, collection «L'évolution de l'humanité », 2011, 509 p., ISBN 978222620 8866, $28 €$.

1 L'ouvrage de Thomas Le Roux, la nuisance en milieu urbain, renvoie à un nouveau champ d'études, l'histoire de l'environnement. Au croisement de l'histoire urbaine, de l'histoire des savoirs (hygiène publique, chimie industrielle), des sciences et des techniques (de la proto-industrialisation à l'industrialisation proprement dite), de l'histoire institutionnelle (la police en premier lieu, mais aussi les sociétés savantes), ce «nouveau " champ d'études a déjà été bien labouré dans les pays voisins (Mary Anderson et Eric Ashby, The Politics of Clean Air, non traduit, ou bien Ulrich Beck, La société du risque, qui a été traduit de l'allemand), mais il donne lieu à des publications récentes (Geneviève Massard-Guilbaud, Histoire de la pollution industrielle en France au XIXe siècle, notamment), parmi lesquelles cet ouvrage fera date.

2 Toutes ces études ont très nettement souligné l'importance fondatrice du décret impérial du 15 octobre 1810 sur les établissements industriels insalubres. Mais l'originalité de l'approche de Thomas Le Roux tient au contexte, qu'il appréhende comme période de transition, ce qui permet de bien mettre en perspective l'épisode révolutionnaire, et de souligner notamment le rôle de l'expertise dans la mise en place des politiques de prévention. L'instance scientifique, parallèle et parfois concurrente, très souvent complémentaire, de l'instance administrative, apparaît dans les dernières décennies de l'Ancien Régime, ce qui marque la continuité avec les démarches suivies dans la période post-révolutionnaire. L'autre fil directeur de ce travail, c'est son inscription dans un espace, celui de la capitale au moment où celle-ci va devenir le 
premier pôle industriel français, supplantant les villes du textile - Rouen, Lille, Lyon et les arsenaux.

3 À travers la perception du paysage urbain, du décor souillé de la grande ville, c'est toute la désillusion de la croyance au progrès qui s'inscrit en filigrane. Dans sa progression chronologique, le livre va suivre les étapes, les réalisations et les contradictions de ces manifestes pour l'industrialisation dérégulée ; le symbole étant ce décret de 1810 qui, sous prétexte de réguler les nuisances, va en permettre la généralisation dans la capitale.

La régulation traditionnelle est le fait de la police; elle est guidée par des impératifs de proximité. La police urbaine est une police des rues; éviter les encombrements, nettoyer les immondices, limiter les pestilences et les tapages nocturnes, prévenir surtout les incendies, risque majeur dans les villes modernes. Les moyens à disposition de l'administration sont les enquêtes de voisinage (commodo et incommodo), l'autorisation préalable, et l'éloignement des activités reconnues insalubres ou dangereuses (par le jeu intra-muros, extra-muros). C'est particulièrement le cas des activités qui utilisent des fours, ou qui présentent un risque de déflagration (poudreries, artifices). Les tanneries qui sont liées aux cours d'eau.

5 Ces modes traditionnels de régulation n'ont guère évolué sur un siècle, depuis l'ère du traité de Delamare. La nouveauté dans la dernière décennie de l'Ancien Régime vient du recours à une expertise scientifique, à côté de la procédure administrative traditionnelle. Cette expertise s'appuie sur les médecins et les scientifiques, qui énoncent des principes différents, mais complémentaires; pour les uns, comme Parmentier, la défense de la présence des ateliers intra-muros s'appuie sur l'idée que c'est dans les lieux les plus industriels que l'air est le plus salubre (l'industrie nous débarrasse des miasmes, des immondices, et des gravats). Pour d'autres, s'installe le préjugé que les ateliers artisanaux sont dangereux et insalubres en soi, et doivent être à ce titre éloignés le plus possible du centre des villes et regroupés dans des lieux spécifiques. Mais il n'y a pas séparation d'avec l'industrie. En ces lieux spécifiques, il serait plus aisé de pratiquer un traitement chimique. C'est le courant hygiéniste, qui participe par exemple au même moment de l'éloignement des cimetières de la proximité des lieux habités. Cadet de Vaux représente parfaitement ce courant hygiéniste. Par son intermédiaire, et par le biais de ce courant hygiéniste, les pharmaciens se constituent en experts scientifiques.

6 Les deux tendances se retrouvent dans l'entourage du lieutenant de police Lenoir. Avec le départ de ce dernier en 1785 , la léthargie des pouvoirs publics contribue à la multiplication intra-muros des activités artisanales sans autorisation préalable, tendance qui va se développer pleinement à l'époque de la Révolution, profitant de la réorganisation des compétences policières, et de la dérégulation générale des activités économiques. C'est dans cette « dynamique économique » que la remise en question des régulations traditionnelles va surtout puiser ses arguments; industrie des acides (site de Javel), papiers peints, dérivés des produits animaux pour les colles et les vernis, emploi du charbon de terre (pompes à feu de Chaillot, du Gros-Caillou). Comme il est dit p. 161 : « les activités anciennes qui reposaient sur la putréfaction continuaient d'être régulées selon d'anciennes pratiques, tandis que les activités innovantes et modernes, qui pouvaient fonder la puissance du Royaume, furent encouragées en vertu d'impératifs économiques ». 
7 On observe le passage progressif des activités artisanales (fours des boulangers, forges des serruriers et des maréchaux-ferrants, tueries et fonderies de suif des bouchers) vers des établissements plus grands, producteurs de nuisances d'un autre type. La transition se fit bien souvent par un changement d'échelle, et un réaménagement des espaces, des activités artisanales traditionnelles; l'installation de la grande triperie de l'île aux cygnes, avec toutes les activités dérivées, se fait à proximité de nombreuses activités industrielles modernes (acides, pompe à feu) à Javel ou au Gros-Caillou.

8 Les années 1789-1795 sont toujours tiraillées entre les deux dynamiques inverses (disons pour aller vite la «dynamique réglementaire» et la «dynamique économique »). Mais c'est très nettement la deuxième tendance qui l'emporte, avec le soutien du pouvoir politique. La vacance institutionnelle est mise à profit pour contourner les réglementations qui portent sur le bruit, ou sur l'abattage domestique des animaux, par exemple. Tandis que l'entrée en guerre transforme la capitale en arsenal de la République, avec toutes les activités afférentes; forge à fusils, récolte du salpêtre, traité ensuite dans les chaudières de raffineurs et des brasseurs, et surtout poudrières, établissement les plus dangereux. Les risques d'incendies se multiplient, le plus grave et le plus spectaculaire étant l'explosion de la poudrerie de Grenelle le 14 fructidor an II (31 août 1794). Il y eut 550 morts et 840 blessés.

9 Cette catastrophe marque un peu la fin d'une époque, et le retour à des activités plus régulées. Sous le Directoire, c'est le Bureau central du département de la Seine qui va gérer le pouvoir de régulation. Il s'efforce de renouer avec l'esprit réglementaire de l'Ancien Régime; restauration des enquêtes de voisinage (commodo et incommodo); éradication des fours à plâtre dans Paris, restrictions à la présence de barques et de chantiers le long de la Seine, tentatives pour concentrer le traitement des déchets animaux. Les attributions échoient sous le Consulat à la préfecture de Police ; toutefois, il y eut un changement dans l'état d'esprit, et une plus grande harmonisation entre les instances régulatrices et le ministère de l'Intérieur. La personnalité de Chaptal domine la période, et l'esprit industrialiste triomphe. La nouveauté réside dans la création du Conseil de salubrité en 1802, dans la droite ligne de l'expertise scientifique de la fin de l'Ancien Régime ; « le pouvoir se tournait maintenant beaucoup plus volontiers vers les savants, et notamment les pharmaciens-chimistes, que vers les commissaires de police, pour estimer les nuisances de l'industrie, afin d'obtenir des expertises qui se voulaient plus scientifiques»( notamment l'action du Conseil de salubrité, davantage que les médecins, dont la profession est alors en pleine recomposition.

10 Les établissements industriels vont se multiplier au centre de Paris, fonctionnant cette fois avec les dérivés de la chimie (la soude artificielle, le chlore, l'acide sulfurique). Les hommes de science comme Chaptal, ou bien Darcet, Fourcroy, Berthollet, sont aussi des entrepreneurs, et des hommes politiques. Science, pouvoir et industrie sont fortement intriqués, et l'action du Conseil de salubrité ne peut s'évaluer indépendamment de l'action de la Société d'encouragement à l'industrie nationale, fondée en 1801. Chaptal préside cette dernière, tandis que Guyton-Morveau est le vice-président. C'est devant l'Institut que Chaptal présente en décembre 1804 son rapport sur les établissements industriels insalubres : «Il est donc de première nécessité pour la prospérité des arts qu'on pose enfin des limites qui ne laissent plus rien à l'arbitraire du magistrat, qui tracent au manufacturier le cercle dans lequel il peut exercer son industrie librement 
et sûrement, et qui garantissent au propriétaire voisin qu'il n'y a pas danger ni pour sa santé, ni pour les produits de son sol ».

11 Cet esprit industrialiste assimile l'esprit d'entreprise à l'intérêt collectif, menacé par l'arbitraire policier, les préjugés et l'ignorance de l'opinion publique, la jalousie des voisins et des concurrents. Il postule que la nuisance produite par l'entreprise (fumées acides, ou chargées de particules) ainsi que le risque (chaudières pour les incendies, bruit des marteaux qui peuvent produire des éboulements) ne seront pas surmontés par des interdictions et des limitations, mais par le développement même du progrès technique, à l'image des fumigations pour assainir l'atmosphère préconisées par Guyton-Morveau, ou Vicq d'Azir. Ce sont les « réformes dérégulatrices ».

12 Finalement, il y a une étonnante continuité de ce courant tout à la fois industrialiste et hygiéniste, par-delà les divers épisodes politiques. Il semblerait même qu'il triomphe sous l'Empire et la Restauration, dont on ne retient habituellement que l'aspect "réaction politique ». Toutefois, Thomas Le Roux souligne en conclusion que cette légitimation ne fut pas linéaire, que la victoire ne se fit pas sans résistances. Après la phase de déréglementation sauvage et transitoire qui fut celle des premières années de la Révolution, la création du ministère de la Police générale sous le Directoire (p. 224-225) semble marquer une forme de « restauration régulatrice».

13 La géographie parisienne, dans cette phase de décollage industriel, est vue de façon très fine. Faubourgs industriels contre densification démographique dans les anciennes limites, l'opposition n'est pas aussi tranchée. C'est Paris qui fixe les établissements de l'industrie naissante, les pôles majeurs du développement ultérieur en banlieue sont essentiellement guidés par le relâchement de la pression foncière, et l'axe de la Seine. Les ateliers colonisent les anciens maraîchages, les chantiers de bois, les carrières, les établissements ecclésiastiques désaffectés (on ne se lassera jamais de rappeler que c'est sous le dévôt régime de la Restauration, que le plus grand nombre d'anciens biens nationaux de première origine furent transformés en carrières de pierre, en ateliers de filature, en batteries de chaudières, etc...), Est contre ouest, banlieue contre centre, tout cela se brouille alors; les ateliers s'implantent aussi bien dans la Plaine de Grenelle et à Passy, que dans l'île de la Cité, ou près de la Salpêtrière. On est bien dans une phase de mutation de l'ordre public, avec ce passage entre une police des rues et une planification urbaine globale. Mais on est aussi dans le prolongement de la protoindustrialisation, les nouvelles activités conservant leurs liens étroits avec le grand marché de consommation de proximité.

Ce sont là quelques aperçus de ce travail original, extrêmement documenté, dont les historiens de la décennie révolutionnaire peuvent tirer le plus grand profit par les problématiques nouvelles auxquelles il nous invite. 